ISSN 0103-9954

\title{
DANOS PROVOCADOS PELO FOGO SOBRE A VEGETAÇ̃̃O NATURAL EM UMA FLORESTA PRIMÁRIA NO ESTADO DO ACRE, AMAZÔNIA BRASILEIRA
}

\section{DAMAGES CAUSED BY FIRE ON THE NATURAL VEGETATION IN A PRIMARY FOREST IN ACRE STATE, BRAZILIAN AMAZON}

\author{
Henrique José Borges de Araujo ${ }^{1}$ Luis Cláudio de Oliveira ${ }^{2}$ Sumaia Saldanha de Vasconcelos ${ }^{3}$ \\ Manoel Freire Correia ${ }^{4}$
}

\section{RESUMO}

A emissão do $\mathrm{CO}_{2}$ é uma das principais causas do efeito estufa e as queimadas amazônicas contribuem para isso. Devido à alta umidade retida, a floresta primária amazônica é considerada imune a queimadas, todavia, sob condições climáticas anormais é vulnerável. Este estudo objetiva avaliar os efeitos do fogo, decorrentes de incêndios florestais ocorridos em 2005, sobre uma floresta natural primária no Estado do Acre. Foram monitorados os efeitos do fogo em árvores lenhosas, palmeiras e cipós em três níveis de tamanho: I-DAP $\geq 5 \mathrm{~cm}$; II- $5 \mathrm{~cm}>\mathrm{DAP} \geq 2 \mathrm{~cm}$; e III-DAP $<2 \mathrm{~cm}$ e altura $\geq 1,0 \mathrm{~m}$. Os indivíduos foram avaliados quanto à Condição Geral (níveis I e II), Casca e Copa (Nível I) e brotação. Foram efetuadas cinco avaliações, a primeira em novembro de 2005 e a última em janeiro de 2009. Os resultados mostraram que os indivíduos de menor porte foram os mais impactados e apresentaram as maiores taxas de mortalidade, chegando a $80,1 \%$ no Nível II e decrescendo quanto maior a classe diamétrica, sendo nula (0 \%) nas superiores. Foi observado aumento do número de indivíduos sem dano aparente em todas as classes diamétricas e aumento de $43 \%$ no número de espécies na regeneração, indicando um processo de recuperação da floresta. Foi verificada redução expressiva na diversidade de espécies (15\% no Nível I e 33 \% no Nível II), indicando que a floresta foi modificada quanto à composição florística. Com base nos padrões de danos causados por apenas um incêndio, o caso da área estudada, é esperado que a incidência de novos incêndios, em curtos intervalos insuficientes à recuperação, resulte na irremediável degeneração da floresta.

Palavras-chave: floresta amazônica; queimadas; incêndios florestais.

\section{ABSTRACT}

The emission of $\mathrm{CO}_{2}$ is an important cause of the greenhouse effect and the Amazonian burns contribute to it. Because of the high moisture retained, the primary Amazon forest is considered immune to fire, however, under abnormal climate conditions it is vulnerable. The objective of this study is to evaluate the effects of fire, originated from forest-fires occurred in 2005, in a natural primary forest in the state of Acre, Brazilian Amazon region. The effects of fire on trees, palm trees and lianas were evaluated in three levels of size: I-DBH $\geq 5 \mathrm{~cm} ; \mathrm{II}-5 \mathrm{~cm}>\mathrm{DBH} \geq 2 \mathrm{~cm}$; and III-DBH $<2 \mathrm{~cm}$ and height $\geq 1,0 \mathrm{~m}$. The individuals were evaluated for General Condition (levels I and II), Bark and Cup (Level I) and budding. Five evaluations were made, the first in November 2005 and last in January 2009. The results showed that the smallest subjects were the most impacted ones and showed the highest mortality rates, reaching $80.1 \%$ for Level II and decreasing according to the increasing size of the tree and it is null $(0 \%)$ in higher classes. It was observed a growing number of individuals with no apparent damage in all diameter classes and a $43 \%$ increase in the number of species in regeneration, indicating a recovery process of the forest. It was observed a significant reduction

1. Engenheiro Florestal, MSc., Pesquisador da Empresa Brasileira de Pesquisa Agropecuária, Embrapa Acre, Rodovia BR-364, km 14, CEP 69908-970, Rio Branco (AC). henrique@cpafac.embrapa.br

2. Engenheiro Florestal, MSc., Pesquisador da Empresa Brasileira de Pesquisa Agropecuária, Embrapa Acre, Rodovia BR-364, km 14, CEP 69908-970, Rio Branco (AC). lclaudio@cpafac.embrapa.br

3. Engenheira Agrônoma, MSc., Programa de Pós-graduação em Ciências de Florestas Tropicais, Instituto Nacional de Pesquisas da Amazônia, Av. André Araújo, 2936, CEP 69060-001, Manaus (AM). sumaia_sv@hotmail.com

4. Biólogo, Assistente de Pesquisa da Empresa Brasileira de Pesquisa Agropecuária, Embrapa Acre, Rodovia BR-364, km 14, CEP 69908-970, Rio Branco (AC). freire@cpafac.embrapa.br

Recebido para publicação em 3/03/2010 e aceito em 11/04/2012 
of species diversity (15\% in Level I and $33 \%$ in Level II), showing that the forest was modified in its floristic composition. Based on the significant damage caused by only one fire, the case of study, it is expected the incidence of new fires, at short intervals insufficient for the recovery and it will promote the irremediable degeneration of forest.

Keywords: Amazon forest; Amazonian burns; forest-fires.

\section{INTRODUÇÃO}

Além das agressões antrópicas desmedidas que vem sofrendo, originadas nas décadas de setenta e oitenta pela ocupação econômica (MARGULIS, 2003; FEARNSIDE, 2003; ALENCAR et al., 2004; LAURANCE et al., 2004; FERREIRA et al., 2005), a floresta amazônica é impactada severamente pelas mudanças climáticas globais em curso.

As atividades econômicas têm aumentado drasticamente as taxas de desmatamento na amazônica brasileira e as estimativas indicam que a área total alterada atinge cerca de $15 \%$ da floresta original (IBGE, 2008). A diminuição das chuvas sobre a floresta amazônica, apontada como o resultado dos fenômenos El Nino e do aumento da temperatura de superfície do oceano Atlântico Norte, decorrente do aquecimento global, é uma ameaça, pois com menos chuva é grande o risco de que a mata densa e exuberante, que se espalha por quase sete milhões de quilômetros quadrados na América do Sul, se transforme em uma vegetação mais baixa, rala e seca, cuja aparência fará lembrar as savanas (COX et al., 2000, 2008; BALCH et al., 2008).

Estudos mostram que entre os principais fatores de desequilíbrio global do clima está o aumento da emissão de gases, especialmente o $\mathrm{CO}_{2}$, causadores do efeito estufa (COX et al., 2000; NEPSTAD et al., 2002; FEARNSIDE, 2003; NOBRE et al., 2007; PHILLIPS et al., 2009). As queimadas e incêndios florestais ocorrentes na região amazônica têm contribuído na emissão de $\mathrm{CO}_{2}$, o que leva a um círculo vicioso, em que a emissão deste gás provoca aquecimento e seca (efeito estufa) e propicia condições ambientais ainda mais favoráveis às próprias queimadas e incêndios.

A cobertura florestal amazônica exerce um papel preponderante para a redução da velocidade das mudanças climáticas e na medida em que é removida agrava-se o quadro, pois fica reduzida sua capacidade de guardar e capturar o $\mathrm{CO}_{2}$, além de ocorrer, pela queima da biomassa, o aumento da emissão deste gás para a atmosfera (PHILLIPS et al., 2009). A potencialização do efeito estufa causa nas florestas a estagnação do crescimento das ár- vores, reduzindo o sequestro de carbono, além de aumentar a quantidade de material orgânico morto, que se transforma em combustível e fonte de emissão de gás carbônico (NEPSTAD et al., 2004; BRANDO et al., 2008).

Em razão da alta umidade retida pelos vegetais e ambiente, sobretudo no solo e entorno (raízes, resíduos vegetais e líter), pode-se afirmar que o ecossistema florestal amazônico é dotado de imunidade natural contra queimadas. Todavia, quando exposto a condições climáticas anormais é verificado que esse ecossistema não possui defesas e é bastante vulnerável ao fogo. Foi o caso da grande seca de 2005, atribuída ao aquecimento global (COX et al., 2008; MARENGO et al., 2008a, 2008b), que afetou principalmente a parte sul da Amazônia onde as habituais queimadas fugiram totalmente ao controle e atingiram grandes áreas de florestas primárias.

Na Amazônia, eventos de fogo podem ser de três tipos: a) queimadas originadas de desmatamentos - são resultantes da derrubada e queima da floresta; b) queimadas em áreas desmatadas - são associadas à manutenção e limpeza de pastagens, lavouras e capoeiras; e c) incêndios florestais rasteiros - são oriundos de queimadas que escapam ao controle e invadem florestas primárias ou exploradas para madeira (NEPSTAD et al., 1999).

O ecossistema florestal amazônico, quando impactado por secas e pelo fogo, pode ficar severamente comprometido. Isso se deve em razão de que são poucas as espécies de árvores de florestas tropicais capazes de tolerar o estresse térmico e hídrico e a perturbação provocada, além de afetar também a capacidade de regeneração da floresta, uma vez que plantas jovens em estágio de muda são destruídas e o banco de sementes das gerações futuras é danificado. A maioria das espécies de árvores da Amazônia tem uma casca protetora muito fina para o tamanho do tronco em indivíduos adultos e sua resistência ao fogo é mínima (BARLOW e PERES, 2003).

O presente estudo objetiva avaliar os efeitos do fogo, em termos de danos e de resiliência das árvores, em uma floresta natural primária atingida por incêndio no leste do Estado do Acre. 


\section{MATERIAL E MÉTODOS}

A área do estudo, com ponto central nas coordenadas geográficas S00946'200" e W067'06'300", é localizada no Projeto de Colonização Pedro Peixoto, rodovia BR-364, município de Senador Guiomard, a $110 \mathrm{~km}$ de Rio Branco, capital do Estado do Acre, Amazônia brasileira. É composta por pequenas propriedades que, juntas, possuem 470 hectares de florestas sob manejo florestal madeireiro. O clima é do tipo Aw (Köppen), com três meses de seca, precipitação anual entre 1.800 a $2.000 \mathrm{~mm}$ e temperatura média anual de $24{ }^{\circ} \mathrm{C}$; os solos predominantes são distróficos, com alto teor de argila; a rede de drenagem é constituída na maior parte por pequenos igarapés semiperenes; a topografia é plana; a vegetação é composta pela floresta tropical semiperenifólia, com formações de floresta aberta e floresta densa (BRASIL, 1976).

Nos meses de agosto e setembro de 2005 a área do estudo, onde se localizam os talhões de manejo florestal $(3,6$ hectares cada), foi atingida por incêndios florestais do tipo rasteiro em cerca de $85 \%$ de sua extensão (perto de 400 hectares). Nesta área não se encontraram registros de ocorrência de incêndios no passado e estes não mais ocorreram após o início dos trabalhos deste estudo.

A seca de 2005 foi, até então, em termos históricos, considerada a mais intensa já ocorrida na região amazônica (BROWN et al., 2006; PHILLIPS et al., 2009). Dados da estação meteorológica de Rio Branco (MAPA/Agritempo-INMET), no Acre, mostram que no período de maio a setembro de 2005 houve considerável redução das chuvas, ao mesmo tempo em que a temperatura máxima, exceto nos meses de julho e setembro, manteve-se acima da média no mesmo período (Figura 1).

Foram alocadas na área do estudo 40 parcelas amostrais permanentes de $400 \mathrm{~m}^{2}$ cada, totalizando 1,6 ha (Figura 2).

Os indivíduos existentes nas parcelas amostrais, com predomínio de árvores lenhosas, seguido de palmeiras e cipós, foram monitorados quanto aos efeitos do fogo. Foram efetuadas cinco avaliações em intervalos médios de 10 meses, a saber: $1^{\mathrm{a}}$ avaliação, em novembro de $2005 ; 2^{\mathrm{a}}$ avaliação, em maio de 2006; $3^{\mathrm{a}}$ avaliação, em março de 2007; $4^{\mathrm{a}}$ avaliação, em janeiro de 2008; e $5^{\mathrm{a}}$ avaliação, em janeiro de 2009. O intervalo entre a primeira e a última avaliação totalizou três anos e dois meses.

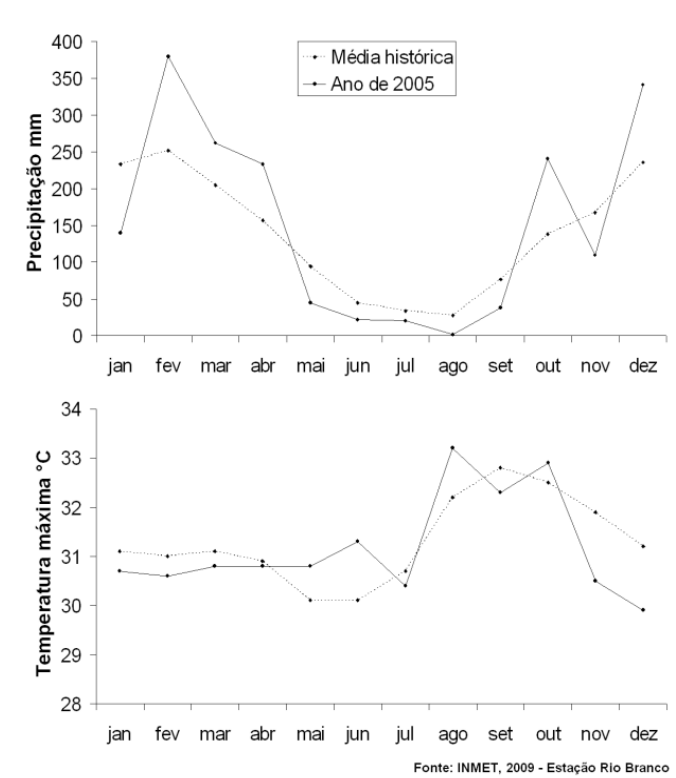

FIGURA 1: Precipitação pluviométrica e temperatura máxima mensal: média histórica e para 2005.

FIGURE 1: Monthly precipitation and maximum temperature: historical average and 2005.

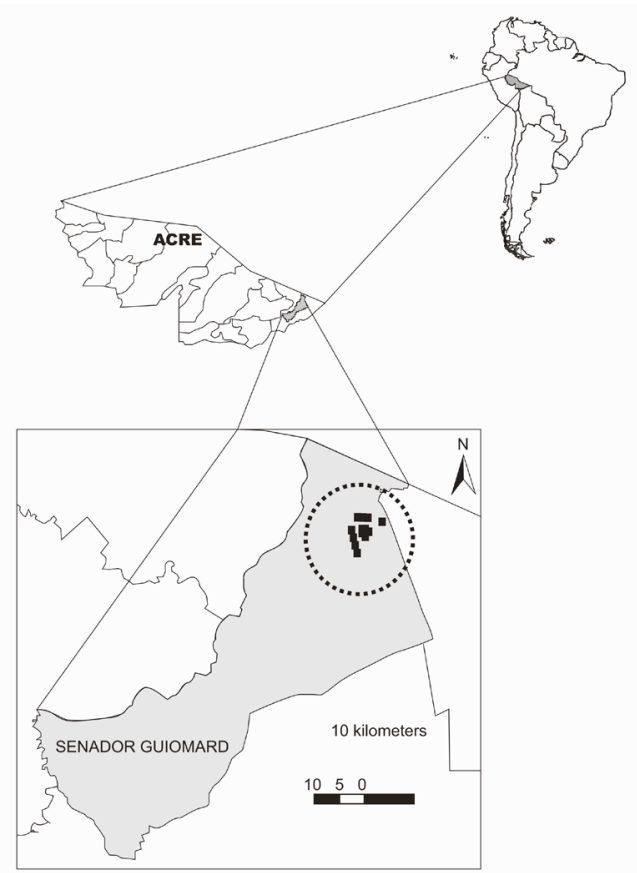

FIGURA 2: Localização da área de estudo no município de Senador Guiomard, Estado do Acre, Brasil, e das parcelas amostrais permanentes (quadrados no círculo pontilhado).

FIGURE 2: Location of study area in the municipal district of Senador Guiomard, State of Acre, Brazil, and the permanent sample plots (squares on dotted circle). 
As parcelas amostrais foram distribuídas sistematicamente pelas áreas queimadas, sendo dispostas duas parcelas a cada cinco talhões (o primeiro talhão foi sorteado), resultando que em oito propriedades foram alocadas quatro parcelas e em quatro duas parcelas amostrais. A avaliação foi efetuada em três níveis de abordagem (tamanho), quais sejam: Iindivíduos com DAP $\geq 5 \mathrm{~cm}$ (parcela com $400 \mathrm{~m}^{2}$ ); II- indivíduos com $5 \mathrm{~cm}>$ DAP $\geq 2 \mathrm{~cm}$ (subparcela com $100 \mathrm{~m}^{2}$ ); e III- regeneração com DAP $<2 \mathrm{~cm}$ e altura $\geq 1,0 \mathrm{~m}$ (subparcela com $25 \mathrm{~m}^{2}$ ) (Figura 3).

Para cada indivíduo ocorrente nos níveis I e II de abordagem, foi feita, além da verificação do dano causado pelo fogo, a identificação da espécie (nome vulgar, fornecido por identificador botânico, sem coleta de material botânico), mensurado o DAP e observada a existência de brotação, sendo esta caracterizada pela emissão de pequenos ramos contendo folhas e situados, normalmente, na base do tronco. Para o Nível III de abordagem (regeneração) não foi efetuada a verificação do dano do fogo, mas apenas a identificação da espécie (nome vulgar) e contagem das plantas presentes.

Os indivíduos ocorrentes no Nível I foram avaliados, por meio de exame visual, em três aspectos: a) Condição Geral: foi verificado o grau de queima geral, considerando os danos do fogo no indivíduo como um todo (casca, tronco, sapopema, copa, ramos, folhagem, etc.); b) Casca: foi verificado o grau de queima da casca, especificamente na região basal da árvore, próximo ao solo onde incidiu o fogo; e c) Copa: foi verificado o efeito do fogo na região foliar da árvore, examinando-se o ressecamento e queda das folhas. Para o Nível II foi avaliado apenas o aspecto Condição Geral.

Para as análises dos resultados da avaliação da Condição Geral do Nível I, foram estabelecidas seis classes diamétricas, a saber: a) $5 \mathrm{~cm} \leq \mathrm{DAP}<10 \mathrm{~cm}$; b) $10 \mathrm{~cm} \leq \mathrm{DAP}<30 \mathrm{~cm}$; c) $30 \mathrm{~cm} \leq \mathrm{DAP}<50 \mathrm{~cm}$; d) $50 \mathrm{~cm} \leq \mathrm{DAP}<70 \mathrm{~cm}$; e) $70 \mathrm{~cm} \leq \mathrm{DAP}<90 \mathrm{~cm}$; e f) $\mathrm{DAP} \geq 90 \mathrm{~cm}$.

$\mathrm{Na}$ avaliação da Condição Geral (níveis I e II), para cada indivíduo foi atribuída a seguinte graduação do dano provocado pelo fogo: 1-Desvitalizado; 2-Alto; 3-Médio; 4-Baixo; e 5-Não atingido. Os critérios de atribuição da graduação dos danos são assim descritos: Desvitalizado - referente a uma condição em que o indivíduo é encontrado totalmente seco, aparentando não mais ter vida; Alto - quando o indivíduo é encontrado quase todo seco, porém, com algumas poucas partes verdes; Médio quando o indivíduo apresenta mais partes secas do que verdes; Baixo - quando o indivíduo apresenta mais partes verdes do que secas; Não atingido quando o indivíduo é encontrado inteiramente verde, sem danos aparentes causados pelo fogo.

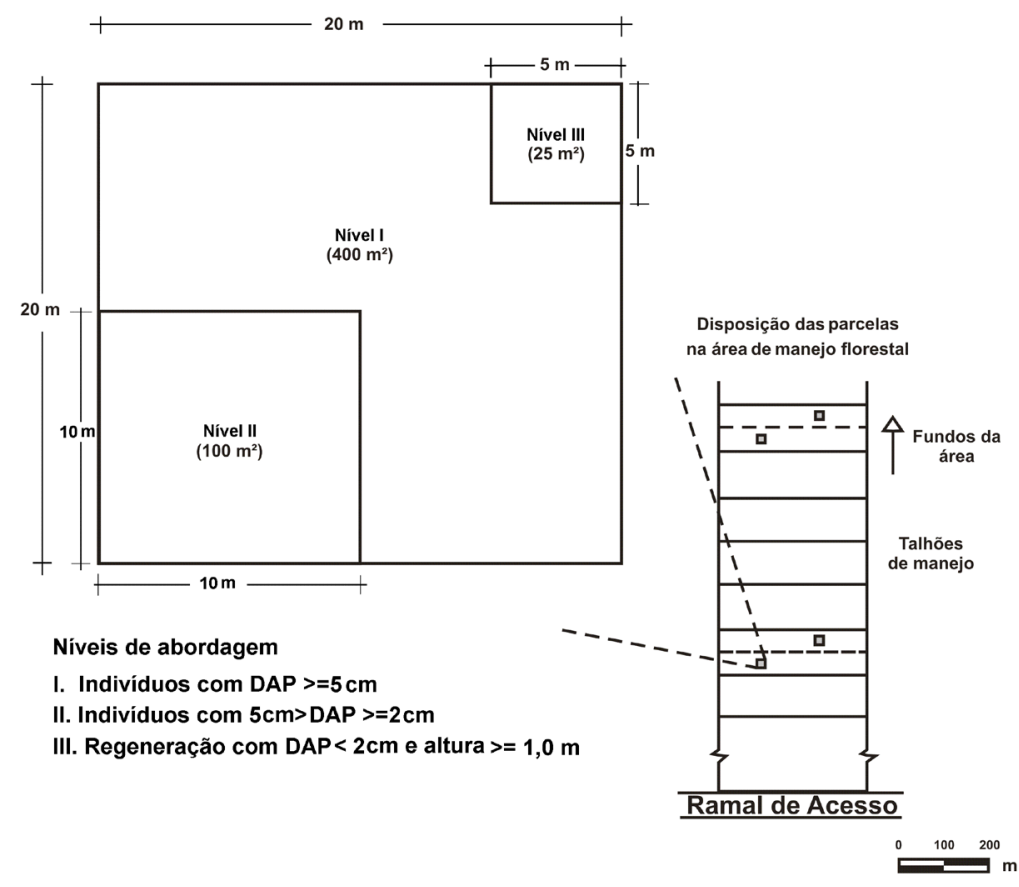

FIGURA 3: Níveis de abordagem e distribuição das parcelas amostrais em uma pequena propriedade componente da área do estudo.

FIGURE 3: Monitored levels of tree size and distribution of plots in a small property in the study area. 
$\mathrm{Na}$ avaliação da Casca, para cada indivíduo foi atribuída a seguinte graduação do dano provocado pelo fogo: 1-Profundo; 2-Severo; 3-Mediano; 4-Leve; e 5-Não atingida. Os critérios de atribuição da graduação dos danos são assim descritos: Profundo - condição em que a casca foi queimada até atingir o lenho; Severo - quando a casca foi muito danificada, porém, sem atingir o lenho; Mediano - quando a casca foi medianamente danificada; Leve - quando a casca foi pouco danificada; Não atingida - quando não houve danos à casca.

$\mathrm{Na}$ avaliação da Copa, para cada indivíduo foi atribuída a seguinte graduação do dano provocado pelo fogo: 1-Copa seca; 2-Muito seca; 3-Medianamente seca; 4-Pouco seca; e 5-Não atingida. Os critérios de atribuição dos danos são assim descritos: Copa seca - condição em que a copa é encontrada totalmente seca ou sem folhas; Muito seca - quando a copa é encontrada com poucas folhas verdes, quase toda seca; Medianamente seca quando a copa é encontrada com mais folhas secas do que verdes; Pouco seca - quando a copa apresenta mais folhas verdes do que secas; Não atingida - quando a folhagem é encontrada toda verde, não havendo danos à copa.

Ressalta-se que nos níveis I e II o número total de indivíduos não variou no decorrer das avaliações efetuadas, pois aqueles não mais encontrados em relação à $1^{\mathrm{a}}$ avaliação receberam a classificação Sem informação e também não foram consideradas as possíveis mudanças de classes diamétricas (ingressos decorridos do crescimento das árvores), mas apenas a medida do DAP da $1^{\mathrm{a}}$ avaliação. Os indivíduos não mais encontrados, na maioria de pequeno porte, foram suprimidos por causas naturais (a exemplo de quedas causadas pelo vento e pelo próprio efeito do fogo) ou não (retirada da madeira, devido à morte, para uso, por exemplo, em benfeitorias da propriedade).

Nas avaliações da Casca e Copa os cipós não foram computados, porque os métodos de avaliação usados às árvores lenhosas e palmeiras não são a eles aplicáveis, pois, em geral, são suspensos e não possuem região basal em contato com o solo bem definida, além de copa difusa e de difícil visualização. Assim, o número total de indivíduos das avaliações da Casca e Copa difere do número total de indivíduos da Condição Geral do Nível I.

As análises estatísticas dos dados foram efetuadas no SAS versão 9.1. Para determinar as associações entre as variáveis qualitativas Espécie, Classe Diamétrica, Condição Geral, Casca e Copa, com evolução da relação Mortalidade (classificações 1-Desvitalizado e Sem informação)/Sobrevivência (classificações 2-Alto; 3-Médio; 4-Baixo; e 5-Não atingido) entre avaliações, foram utilizadas tabelas de contingência (PROC FREQ do SAS 9.1). Para determinar os efeitos das variáveis qualitativas Classe Diamétrica, Casca e Copa, foi ajustado um modelo binário logístico, com técnica de otimização de escores de Fisher (PROC LOGISTIC do SAS 9.1).

\section{RESULTADOS E DISCUSSÃO}

A distribuição do número de indivíduos ocorrentes na $1^{\mathrm{a}}$ avaliação, de acordo com a forma de vida, em cada nível de abordagem, foi a seguinte: Nível I - total de 1856, sendo 1590 (85,7\%) árvores lenhosas, $151(8,1 \%)$ palmeiras e $115(6,2 \%)$ cipós; Nível II - total de 974, sendo $812(83,4 \%)$ árvores lenhosas, 14 (1,4\%) palmeiras e 148 (15,2\%) cipós. Para o Nível III, ocorreram na avaliação inicial um total de 77 espécies, sendo $69(89,6 \%)$ de árvores lenhosas, $2(2,6 \%)$ de palmeiras e $6(7,8 \%)$ de cipós e, na avaliação final $\left(5^{\mathrm{a}}\right)$, um total de 110 espécies, sendo $99(90,0 \%)$ de árvores lenhosas, 4 (3,6 \%) de palmeiras e $7(6,4 \%)$ de cipós.

Aos indivíduos da Condição Geral do Nível I $(\mathrm{DAP} \geq 5 \mathrm{~cm})$, os resultados revelaram um índice bastante elevado de mortalidade (1. Desvitalizado) e um aumento crescente desse dano no decorrer das avaliações realizadas, passando de $556(30,0 \%)$ indivíduos na primeira avaliação para 874 (47,1 \%) na última avaliação. Os indivíduos classificados com danos 2-Alto, 3-Médio e 4-Baixo tiveram um comportamento inverso, ou seja, reduziram significativamente suas participações, o que pode ser explicado pela migração para as classificações extremas. Entretanto, do ponto de vista de resiliência da floresta, o resultado mais importante é em relação ao número de indivíduos sem dano aparente (5Não atingido), em que houve substancial aumento no decorrer das avaliações, passando de 46 (2,5\%) na primeira avaliação para $529(28,5 \%)$ na última avaliação.

Quando observados separadamente por classe diamétrica, os resultados da avaliação da Condição Geral do Nível I, exceto nas classes superiores (DAP $\geq 70 \mathrm{~cm})$, mostraram bastante similaridade aos resultados do conjunto de todas as classes diamétricas, quer seja numa tendência de aumento tanto da mortalidade (1-Desvitalizado), como também do número de indivíduos sem danos 
aparentes (5-Não atingido). As classes diamétricas superiores $(70 \mathrm{~cm} \leq \mathrm{DAP}<90 \mathrm{~cm}$ e $\mathrm{DAP} \geq 90 \mathrm{~cm})$, embora com pouca representatividade amostral (apenas 4 indivíduos em cada classe), não apresentaram mortalidade e sim, no máximo, dano 4-Baixo e, mais ainda, tendendo à recuperação (5-Não atingido).

Em termos absolutos (número total de indivíduos) apenas a classe $5 \mathrm{~cm} \leq \mathrm{DAP}<10 \mathrm{~cm}$ apresen- tou mortalidade (1-Desvitalizado) superior em proporção às demais classes. Nesta classe diamétrica houve tendência de estabilização de crescimento da mortalidade e do número de indivíduos sem danos aparentes (5-Não atingido). Os indivíduos classificados com danos intermediários (2-Alto, 3-Médio, e 4-Baixo) tiveram decréscimos nas participações, enquanto nos suprimidos (Sem informação), acréscimo (Tabela 1).

TABELA 1: Número de dos indivíduos na avaliação da Condição Geral do Nível I (DAP $\geq 5 \mathrm{~cm}$ ) e Nível II $(5 \mathrm{~cm}>\mathrm{DAP} \geq 2 \mathrm{~cm})$ por dano provocado, classe diamétrica e para a sequência de avaliações.

TABLE 1: Number of individuals in evaluation the General Condition of the Level I $(\mathrm{DBH} \geq 5 \mathrm{~cm})$ and Level II $(5 \mathrm{~cm}>\mathrm{DBH} \geq 2 \mathrm{~cm})$ by damage caused, diameter class and the sequence of evaluations.

\begin{tabular}{|c|c|c|c|c|c|c|c|c|c|}
\hline \multirow{2}{*}{ AVAL } & \multirow{2}{*}{$\begin{array}{l}\text { DANO } \\
\text { PROVOCADO }\end{array}$} & \multicolumn{7}{|c|}{ NÍVEL I } & \multirow{2}{*}{ NÍVEL II } \\
\hline & & CLASS 1 & CLASS 2 & CLASS 3 & CLASS 4 & CLASS 5 & CLASS 6 & TOTAL & \\
\hline \multirow[t]{7}{*}{$1^{\mathrm{a}}$} & 1-Desvitalizado & 429 & 118 & 6 & 3 & - & - & 556 & 835 \\
\hline & 2-Alto & 90 & 52 & 4 & 2 & - & - & 148 & 20 \\
\hline & 3-Médio & 53 & 50 & 1 & 1 & 1 & - & 106 & 21 \\
\hline & 4-Baixo & 461 & 442 & 71 & 19 & 3 & 4 & 1000 & 72 \\
\hline & 5-Não atingido & 19 & 23 & 2 & 2 & - & - & 46 & 26 \\
\hline & Sem informação & - & - & - & - & - & - & - & - \\
\hline & TOTAL & 1052 & 685 & 84 & 27 & 4 & 4 & 1856 & 974 \\
\hline \multirow[t]{7}{*}{$2^{\mathrm{a}}$} & 1-Desvitalizado & 560 & 178 & 9 & 6 & - & - & 753 & 866 \\
\hline & 2-Alto & 7 & 6 & - & - & - & - & 13 & 7 \\
\hline & 3-Médio & 35 & 28 & 4 & - & - & - & 67 & 6 \\
\hline & 4-Baixo & 377 & 394 & 58 & 16 & 4 & 1 & 850 & 35 \\
\hline & 5-Não atingido & 62 & 75 & 13 & 4 & - & 3 & 157 & 50 \\
\hline & Sem informação & 11 & 4 & - & 1 & - & - & 16 & 10 \\
\hline & TOTAL & 1052 & 685 & 84 & 27 & 4 & 4 & 1856 & 974 \\
\hline \multirow[t]{7}{*}{$3^{a}$} & 1-Desvitalizado & 539 & 224 & 9 & 8 & - & - & 780 & 584 \\
\hline & 2-Alto & 109 & 20 & 2 & 1 & - & - & 132 & 235 \\
\hline & 3-Médio & 14 & 8 & 2 & - & - & - & 24 & 9 \\
\hline & 4-Baixo & 124 & 114 & 13 & 2 & 1 & - & 254 & 11 \\
\hline & 5-Não atingido & 231 & 305 & 57 & 15 & 3 & 4 & 615 & 63 \\
\hline & Sem informação & 35 & 14 & 1 & 1 & - & - & 51 & 72 \\
\hline & TOTAL & 1052 & 685 & 84 & 27 & 4 & 4 & 1856 & 974 \\
\hline \multirow[t]{7}{*}{$4^{a}$} & 1-Desvitalizado & 531 & 229 & 13 & 8 & - & - & 781 & 534 \\
\hline & 2-Alto & 91 & 14 & 1 & - & - & - & 106 & 202 \\
\hline & 3-Médio & 7 & 5 & 1 & - & - & - & 13 & 4 \\
\hline & 4-Baixo & 51 & 68 & 6 & 1 & - & - & 126 & 7 \\
\hline & 5-Não atingido & 264 & 309 & 60 & 14 & 4 & 4 & 655 & 68 \\
\hline & Sem informação & 108 & 60 & 3 & 4 & - & - & 175 & 159 \\
\hline & TOTAL & 1052 & 685 & 84 & 27 & 4 & 4 & 1856 & 974 \\
\hline \multirow[t]{7}{*}{$5^{\mathrm{a}}$} & 1-Desvitalizado & 583 & 261 & 19 & 11 & - & - & 874 & 545 \\
\hline & 2-Alto & 23 & 4 & - & - & - & - & 27 & 89 \\
\hline & 3-Médio & 5 & 3 & - & - & - & - & 8 & 32 \\
\hline & 4-Baixo & 77 & 79 & 11 & - & 2 & - & 169 & 15 \\
\hline & 5-Não atingido & 196 & 268 & 47 & 12 & 2 & 4 & 529 & 58 \\
\hline & Sem informação & 168 & 70 & 7 & 4 & - & - & 249 & 235 \\
\hline & TOTAL & 1052 & 685 & 84 & 27 & 4 & 4 & 1856 & 974 \\
\hline
\end{tabular}

Em que: $A V A L=$ Avaliação, CLASS 1: $5 \mathrm{~cm} \leq$ DAP $<10 \mathrm{~cm}$, CLASS 2: $10 \mathrm{~cm} \leq$ DAP $<30 \mathrm{~cm}$, CLASS 3: $30 \mathrm{~cm} \leq$ DAP $<50$ $\mathrm{cm}$, CLASS 4: $50 \mathrm{~cm} \leq$ DAP $<70 \mathrm{~cm}$, CLASS 5: $70 \mathrm{~cm} \leq$ DAP $<90 \mathrm{~cm}$, CLASSE 6: DAP $\geq 90 \mathrm{~cm}$. 
Na $1^{\mathrm{a}}$ avaliação do Nível I, foi identificado em campo um total de 177 espécies, sendo 160 de árvores lenhosas, 10 de palmeiras e 7 de cipós. Na $5^{\text {a }}$ avaliação do Nível I, já excluídos os indivíduos mortos (1-Desvitalizado) e suprimidos (Sem informação), foi identificado um total de 150 espécies, das quais 134 foram árvores lenhosas, 10 palmeiras e 6 cipós. Deste modo, houve uma redução de 27 (15\%) espécies na diversidade existente originalmente nas parcelas amostrais, sendo que 26 são espécies de árvores lenhosas e 1 de cipó.

Para o Nível II ( $5 \mathrm{~cm}>$ DAP $\geq 2 \mathrm{~cm})$, que na $1^{\mathrm{a}}$ avaliação totalizou 974 indivíduos, os resultados revelaram um índice de mortalidade (1. Desvitalizado) ainda mais elevado do que a classe diamétrica, mais impactada do Nível I $(5 \mathrm{~cm} \leq \mathrm{DAP}<10 \mathrm{~cm})$, no entanto, ao contrário do Nível I, foi verificado decréscimo no decorrer do monitoramento, passando de 835 indivíduos $(85,7 \%)$ na primeira avaliação para $545(56,0 \%)$ na última avaliação. Os indivíduos classificados com danos 2-Alto e 3-Médio aumentam em frequência, sendo os primeiros com maior intensidade. Aqueles com dano 4-Baixo reduziram e os 5-Não atingido aumentaram em frequência. Neste nível de abordagem, o número de indivíduos suprimidos (Sem informação) teve expressivo crescimento, sendo que na última avaliação 235 indivíduos $(24,1 \%)$ presentes na primeira avaliação não mais foram encontrados. $\mathrm{O}$ fato de ter havido expressivo crescimento do número de indivíduos suprimidos (Sem informação), aliado à evolução crescente dos percentuais dessa classificação, sugere que o decréscimo verificado da mortalidade (1-Desvitalizado) pode ser explicado pela migração para classificação Sem informação.

$\mathrm{Na} 1^{\mathrm{a}}$ avaliação do Nível II foi identificado em campo um total de 98 espécies, sendo 89 de árvores lenhosas, 3 de palmeiras e 6 de cipós. Na $5^{\text {a }}$ avaliação do Nível II, já excluídos os indivíduos mortos (1-Desvitalizado) e suprimidos (Sem informação), foi identificado um total de 66 espécies, das quais 62 foram árvores lenhosas, 2 palmeiras e 2 cipós. Deste modo, houve uma redução de 32 (33 \%) espécies na diversidade existente originalmente nas parcelas amostrais, sendo que 27 são espécies de árvores lenhosas, 1 de palmeira e 4 de cipós.

Para o Nível III de abordagem (regeneração com $\mathrm{DAP}<2 \mathrm{~cm}$ e altura $\geq 1,0 \mathrm{~m}$ ), os resultados revelaram, em 40 subparcelas de $25 \mathrm{~m}^{2}$, a ocorrência de 77 espécies na primeira avaliação (correspondente à $2^{\mathrm{a}}$ avaliação do monitoramento, dado que a regeneração encontrava-se destruída na $1^{\text {a }}$ avaliação) e de 110 espécies regenerantes na última avaliação $\left(5^{\mathrm{a}}\right)$, representando um acréscimo de $43 \%$ no número de espécies.

Na avaliação da Casca, efetuada no Nível I $(\mathrm{DAP} \geq 5 \mathrm{~cm}$ ), foi verificado no decorrer das avaliações, exceto entre a $4^{\mathrm{a}}$ e a $5^{\mathrm{a}}$ avaliação de 4-Leve, um decréscimo generalizado do número de indivíduos nas quatro primeiras classificações de danos (1-Profundo, 2-Severo, 3-Mediano e 4-Leve), sendo que o decréscimo do dano máximo (1-Profundo) foi pouco acentuado. Ao contrário, para as classificações de danos 5-Não atingida e Sem informação, houve, exceto entre a $4^{\mathrm{a}}$ e a $5^{\mathrm{a}}$ avaliação de 5-Não atingida, crescimento da participação do número de indivíduos (Tabela 2).

Ainda que tenha havido aumento expressivo do número de indivíduos Sem informação, passando de $14(0,8 \%)$ na $2^{\mathrm{a}}$ avaliação para $222(12,8 \%)$ na $5^{\mathrm{a}}$ avaliação, o que está associado à mortalidade (indivíduos suprimidos), pode-se afirmar, com relativa clareza, que houve ao longo do monitoramento uma recuperação dos indivíduos remanescentes dos danos acometidos pelo fogo na casca. A distribuição do número de indivíduos separadamente por classe diamétrica no decorrer das avaliações da Casca mostra, de modo similar à distribuição do total do número de indivíduos, uma tendência generalizada, exceto nas classes superiores, de decréscimo das participações do número de indivíduos danificados (1-Profundo, 2-Severo, 3-Mediano e 4-Leve) e crescimento do número de indivíduos sem dano aparente e dos Sem informação.

$\mathrm{Na}$ avaliação da Copa, efetuada no Nível I (DAP $\geq 5 \mathrm{~cm}$ ), foi verificado, no decorrer das avaliações, um crescimento do número de indivíduos na classificação de dano máximo (1-Copa Seca). Na classificação sem dano aparente (5-Não atingida), houve crescimento do número de indivíduos da $1^{\text {a }}$ para a $2^{\mathrm{a}}$ avaliação e decréscimo nas demais avaliações. As classificações intermediárias (2-Muito seca, 3-Medianamente seca e 4-Pouco) apresentaram decréscimo generalizado na participação do número de indivíduos. O número de indivíduos Sem informação aumentou consideravelmente no decorrer das avaliações, atingindo o total de 215 (12,3\%) na última avaliação. Na avaliação da Copa houve, desde a $1^{\mathrm{a}}$ avaliação, um certo equilíbrio entre o número de indivíduos com dano máximo (1-Copa seca) e o com dano mínimo (5-Não atingida), com ligeira vantagem para os primeiros. Quando comparado com os danos na casca, esse resultado sugere que a copa das árvores possui maior susceptibilidade e maior tempo de recuperação aos efeitos do fogo (Tabela 3). 
TABELA 2: Número de indivíduos na avaliação da casca por dano provocado, classe diamétrica e para a sequência de avaliações.

TABLE 2: Number of individuals in the evaluation of bark by damage caused, diameter class and the sequence of evaluations.

\begin{tabular}{|c|c|c|c|c|c|c|c|c|}
\hline AVAL & DANO PROVOCADO & CLASS 1 & CLASS 2 & CLASS 3 & CLASS 4 & CLASS 5 & CLASS 6 & TOTAL \\
\hline \multirow[t]{7}{*}{$1^{\mathrm{a}}$} & 1-Profundo & 81 & 27 & 1 & 3 & - & - & 112 \\
\hline & 2-Severo & 186 & 114 & 5 & 4 & - & - & 309 \\
\hline & 3-Mediano & 319 & 158 & 22 & 5 & 2 & - & 506 \\
\hline & 4-Leve & 349 & 345 & 54 & 13 & 2 & 3 & 766 \\
\hline & 5-Não atingida & 18 & 25 & 2 & 2 & - & 1 & 48 \\
\hline & Sem informação & - & - & - & - & - & - & - \\
\hline & TOTAL & 953 & 669 & 84 & 27 & 4 & 4 & 1741 \\
\hline \multirow[t]{7}{*}{$2^{\mathrm{a}}$} & 1-Profundo & 73 & 23 & 2 & 3 & - & - & 101 \\
\hline & 2-Severo & 146 & 59 & 2 & 2 & - & - & 209 \\
\hline & 3-Mediano & 264 & 93 & 8 & 2 & - & - & 367 \\
\hline & 4-Leve & 399 & 413 & 56 & 14 & 4 & 1 & 887 \\
\hline & 5-Não atingida & 61 & 78 & 16 & 5 & - & 3 & 163 \\
\hline & Sem informação & 10 & 3 & - & 1 & - & - & 14 \\
\hline & TOTAL & 953 & 669 & 84 & 27 & 4 & 4 & 1741 \\
\hline \multirow[t]{7}{*}{$3^{\mathrm{a}}$} & 1-Profundo & 72 & 23 & 2 & 2 & - & - & 99 \\
\hline & 2-Severo & 137 & 56 & 2 & 2 & - & - & 197 \\
\hline & 3-Mediano & 257 & 88 & 7 & 2 & - & - & 354 \\
\hline & 4-Leve & 243 & 194 & 16 & 5 & 1 & - & 459 \\
\hline & 5-Não atingida & 214 & 298 & 56 & 15 & 3 & 4 & 590 \\
\hline & Sem informação & 30 & 10 & 1 & 1 & - & - & 42 \\
\hline & TOTAL & 953 & 669 & 84 & 27 & 4 & 4 & 1741 \\
\hline \multirow[t]{7}{*}{$4^{a}$} & 1-Profundo & 65 & 22 & 1 & 2 & - & - & 90 \\
\hline & 2-Severo & 116 & 45 & 2 & 2 & - & - & 165 \\
\hline & 3-Mediano & 237 & 76 & 6 & 2 & - & - & 321 \\
\hline & 4-Leve & 190 & 165 & 13 & 3 & - & - & 371 \\
\hline & 5-Não atingida & 251 & 305 & 59 & 14 & 4 & 4 & 637 \\
\hline & Sem informação & 94 & 56 & 3 & 4 & - & - & 157 \\
\hline & TOTAL & 953 & 669 & 84 & 27 & 4 & 4 & 1741 \\
\hline \multirow[t]{7}{*}{$5^{\mathrm{a}}$} & 1-Profundo & 67 & 23 & 1 & 2 & - & - & 93 \\
\hline & 2-Severo & 108 & 45 & 1 & 2 & - & - & 156 \\
\hline & 3-Mediano & 209 & 76 & 7 & 2 & - & - & 294 \\
\hline & 4-Leve & 241 & 197 & 19 & 5 & 1 & - & 463 \\
\hline & 5-Não atingida & 185 & 260 & 49 & 12 & 3 & 4 & 513 \\
\hline & Sem informação & 143 & 68 & 7 & 4 & - & - & 222 \\
\hline & TOTAL & 953 & 669 & 84 & 27 & 4 & 4 & 1741 \\
\hline
\end{tabular}

Em que: $A V A L=$ Avaliação, CLASS 1: $5 \mathrm{~cm} \leq \mathrm{DAP}<10 \mathrm{~cm}$, CLASS 2: $10 \mathrm{~cm} \leq \mathrm{DAP}<30 \mathrm{~cm}$, CLASS 3: $30 \mathrm{~cm} \leq$ DAP $<50$ $\mathrm{cm}$, CLASS 4: $50 \mathrm{~cm} \leq \mathrm{DAP}<70 \mathrm{~cm}$, CLASS 5: $70 \mathrm{~cm} \leq \mathrm{DAP}<90 \mathrm{~cm}$, CLASS 6: DAP $\geq 90 \mathrm{~cm}$; Não estão computados os cipós.

A distribuição por classe diamétrica na avaliação da Copa mostra, de modo similar ao total dos indivíduos, um crescimento generalizado, exceto nas classes superiores em que não houve danos, do número de indivíduos com dano máximo (1-Copa Seca) tendendo ao decréscimo dos sem danos aparentes (5-Não atingida). Exceto na classe diamétrica $5 \mathrm{~cm} \leq \mathrm{DAP}<10 \mathrm{~cm}$ e nas superiores, os indivíduos sem danos sempre predominaram em proporções maiores.
Os resultados da Tabela 4 mostram que há associações estatísticas significativas entre a relação Mortalidade (1-Desvitalizado e Sem informação)/Sobrevivência (2-Alto; 3-Médio; 4-Baixo; e 5-Não atingido) e as variáveis qualitativas Condição Geral, Casca e Copa até a $5^{\text {a }}$ avaliação, indicando que os efeitos do fogo sobre a casca e copa, com respeito à mortalidade, tendem a se manter no tempo, em função da baixa capacidade de recuperação dos indivíduos atingidos. Espécies 
TABELA 3: Número de indivíduos na avaliação da copa por dano provocado, classe diamétrica e para a sequência de avaliações.

TABLE 3: Number of individuals in the evaluation of cup by damage caused, diameter class and the sequence of evaluations.

\begin{tabular}{|c|c|c|c|c|c|c|c|c|}
\hline AVAL & DANO PROVOCADO & CLASS 1 & CLASS 2 & CLASS 3 & CLASS 4 & CLASS 5 & CLASS 6 & TOTAL \\
\hline \multirow[t]{7}{*}{$1^{\mathrm{a}}$} & 1-Copa seca & 426 & 135 & 8 & 4 & - & - & 573 \\
\hline & 2-Muito seca & 59 & 28 & 2 & 1 & - & - & 90 \\
\hline & 3-Medianamente seca & 66 & 43 & 3 & 1 & - & - & 113 \\
\hline & 4-Pouco seca & 195 & 165 & 21 & 4 & - & - & 385 \\
\hline & 5-Não atingida & 207 & 298 & 50 & 17 & 4 & 4 & 580 \\
\hline & Sem informação & - & - & - & - & - & - & - \\
\hline & TOTAL & 953 & 669 & 84 & 27 & 4 & 4 & 1741 \\
\hline \multirow[t]{7}{*}{$2^{a}$} & 1-Copa seca & 505 & 174 & 9 & 6 & - & - & 694 \\
\hline & 2-Muito seca & 7 & 5 & 2 & - & - & - & 14 \\
\hline & 3-Medianamente seca & 16 & 9 & 2 & - & - & - & 27 \\
\hline & 4-Pouco seca & 58 & 41 & - & - & - & - & 99 \\
\hline & 5-Não atingida & 356 & 437 & 71 & 20 & 4 & 4 & 892 \\
\hline & Sem informação & 11 & 3 & - & 1 & - & - & 15 \\
\hline & TOTAL & 953 & 669 & 84 & 27 & 4 & 4 & 1741 \\
\hline \multirow[t]{7}{*}{$3^{\mathrm{a}}$} & 1-Copa seca & 565 & 230 & 10 & 6 & - & - & 811 \\
\hline & 2-Muito seca & 7 & 2 & 1 & 1 & - & - & 11 \\
\hline & 3-Medianamente seca & 13 & 10 & 1 & - & - & - & 24 \\
\hline & 4-Pouco seca & 12 & 14 & 1 & - & - & - & 27 \\
\hline & 5-Não atingida & 305 & 387 & 68 & 17 & 4 & 4 & 785 \\
\hline & Sem informação & 51 & 26 & 3 & 3 & - & - & 83 \\
\hline & $\begin{array}{ll}\text { TOTAL } \\
\end{array}$ & 953 & 669 & 84 & 27 & 4 & 4 & 1741 \\
\hline \multirow[t]{7}{*}{$4^{\mathrm{a}}$} & 1-Copa seca & 558 & 239 & 15 & 8 & - & - & 820 \\
\hline & 2-Muito seca & 3 & 1 & - & - & - & - & 4 \\
\hline & 3-Medianamente seca & 3 & 3 & - & - & - & - & 6 \\
\hline & 4-Pouco seca & 5 & 8 & - & - & - & - & 13 \\
\hline & 5-Não atingida & 290 & 360 & 66 & 15 & 4 & 4 & 739 \\
\hline & Sem informação & 94 & 58 & 3 & 4 & - & - & 159 \\
\hline & TOTAL & 953 & 669 & 84 & 27 & 4 & 4 & 1741 \\
\hline \multirow[t]{7}{*}{$5^{\mathrm{a}}$} & 1-Copa seca & 552 & 264 & 20 & 11 & - & - & 847 \\
\hline & 2-Muito seca & 2 & 1 & - & - & - & - & 3 \\
\hline & 3-Medianamente seca & 2 & 1 & - & - & - & - & 3 \\
\hline & 4-Pouco seca & 7 & 6 & 1 & - & - & - & 14 \\
\hline & 5-Não atingida & 254 & 329 & 56 & 12 & 4 & 4 & 659 \\
\hline & Sem informação & 136 & 68 & 7 & 4 & - & - & 215 \\
\hline & TOTAL & 953 & 669 & 84 & 27 & 4 & 4 & 1741 \\
\hline
\end{tabular}

Em que: CLASS 1: $5 \mathrm{~cm} \leq$ DAP $<10 \mathrm{~cm}$, CLASS 2: $10 \mathrm{~cm} \leq$ DAP $<30 \mathrm{~cm}$, CLASS 3: $30 \mathrm{~cm} \leq$ DAP $<50 \mathrm{~cm}$, CLASS 4: 50 $\mathrm{cm} \leq \mathrm{DAP}<70 \mathrm{~cm}$, CLASS 5: $70 \mathrm{~cm} \leq \mathrm{DAP}<90 \mathrm{~cm}$, CLASS 6: DAP $\geq 90 \mathrm{~cm}$; Não estão computados os cipós.

e Mortalidade/Sobrevivência mostram uma associação significativa (Gl: 176; $\mathrm{X}^{2}: 298,49 ; \mathrm{p}<$ 0,001), o que indica que as espécies são afetadas de maneira diferente pelo fogo. Classe Diamétrica e Mortalidade/Sobrevivência estão associadas significativamente (Gl: 5; $\mathrm{X}^{2}$ : 95,91; $\left.\mathrm{p}<0,0001\right)$, demonstrando que árvores maiores são menos afetadas pelo fogo.

O modelo logístico (binário logístico) ajustado para determinar os efeitos das variáveis Classe
Diamétrica, Casca e Copa (Classe Diamétrica $=$ $0(5 \mathrm{~cm} \leq \mathrm{DAP}<10 \mathrm{~cm}), 1(10 \mathrm{~cm} \leq \mathrm{DAP}<30 \mathrm{~cm})$, $2(30 \mathrm{~cm} \leq$ DAP $<50 \mathrm{~cm}), 3(50 \mathrm{~cm} \leq$ DAP $<70 \mathrm{~cm})$, $4(70 \mathrm{~cm} \leq \mathrm{DAP}<90 \mathrm{~cm}), 5(\mathrm{DAP} \geq 90 \mathrm{~cm})$; Casca = 1 (1-Profundo), 2 (2-Severo), 3 (3-Mediano), 4 (4-Leve), 5 (5-Não atingida); Copa = 1 (1-Copa seca), 2 (2-Muito seca), 3 (3-Medianamente seca), 4 (4-Pouco seca), 5 (5-Não atingida)), sobre a probabilidade de morte das árvores, com base em 953 árvores mortas e 680 vivas, satisfez o 
TABELA 4: Associação (Pearson $\mathrm{X}^{2}$ ) entre mortalidade/sobrevivência por variável, avaliação e graduação de dano.

TABLE 4: Association (Pearson $\mathrm{X}^{2}$ ), between mortality/survival by variable, evaluation and damage graduation.

\begin{tabular}{|c|c|c|c|c|c|}
\hline VARIÁVEL & $1^{\mathrm{a}}$ AVALIAÇÃO & $2^{\mathrm{a}}$ AVALIAÇÃO & $3^{\text {a }}$ AVALIAÇÃO & $4^{\mathrm{a}}$ AVALIAÇÃO & $5^{\mathrm{a}}$ AVALIAÇÃO \\
\hline Condição Geral & $\begin{array}{l}\mathrm{Gl}=4 \\
\mathrm{X}^{2}=283,15 \\
\mathrm{p}<.0001\end{array}$ & $\begin{array}{l}\mathrm{Gl}=4 \\
\mathrm{X}^{2}=468,56 \\
\mathrm{p}<.0001\end{array}$ & $\begin{array}{l}\mathrm{Gl}=4 \\
\mathrm{X}^{2}=613,28 \\
\mathrm{p}<.0001\end{array}$ & $\begin{array}{l}\text { Gl } 4 \\
\mathrm{X}^{2}=171,05 \\
\mathrm{p}<.0001\end{array}$ & $\begin{array}{l}\text { Gl } 4 \\
X^{2}=1607,00 \\
p<.0001\end{array}$ \\
\hline Casca & $\begin{array}{l}\mathrm{Gl}=4 \\
\mathrm{X}^{2}=195,46 \\
\mathrm{p}<.0001\end{array}$ & $\begin{array}{l}\mathrm{Gl}=4 \\
\mathrm{X}^{2}=339,79 \\
\mathrm{p}<.0001\end{array}$ & $\begin{array}{l}\mathrm{Gl}=4 \\
\mathrm{X}^{2}=489,60 \\
\mathrm{p}<.0001\end{array}$ & $\begin{array}{l}\mathrm{Gl}=4 \\
\mathrm{X}^{2}=155,40 \\
\mathrm{p}<.0001\end{array}$ & $\begin{array}{l}\mathrm{Gl}=4 \\
\mathrm{X}^{2}=956,36 \\
\mathrm{p}<.0001\end{array}$ \\
\hline Copa & $\begin{array}{l}\mathrm{Gl}=4 \\
\mathrm{X}^{2}=280,07 \\
\mathrm{p}<.0001\end{array}$ & $\begin{array}{l}\mathrm{Gl}=4 \\
\mathrm{X}^{2}=437,10 \\
\mathrm{p}<.0001\end{array}$ & $\begin{array}{l}\mathrm{Gl}=4 \\
\mathrm{X}^{2}=574,37 \\
\mathrm{p}<.0001\end{array}$ & $\begin{array}{l}\mathrm{Gl}=4 \\
\mathrm{X}^{2}=161,65 \\
\mathrm{p}<.0001\end{array}$ & $\begin{array}{l}\mathrm{Gl}=4 \\
\mathrm{X}^{2}=1343,92 \\
\mathrm{p}<.0001\end{array}$ \\
\hline
\end{tabular}

Em que: Valores de $\mathrm{p}<0.05$ indicam associações significativas estatisticamente.

critério de convergência e é significativo $(\mathrm{Gl}=3$; Wald $=252,58 ;<.0001)$. As estimativas de máxima verossimilhança para Classe diamétrica $(\mathrm{Gl}=1$; Wald $\left.\mathrm{X}^{2}=28,73 ; \mathrm{p}<0,0001\right)$ e Copa $(\mathrm{Gl}=1$; Wald $\left.\mathrm{X}^{2}=73,79 ; \mathrm{p}<0,0001\right)$ foram $-0,4461$ e $-0,4376$, respectivamente.

O modelo logístico ajustado foi: Log $\rho / 1-\rho=2,5559-0,4461 *$ Classe Diamétrica $0,4376 *$ Copa. O modelo indica o efeito de cada variável explanatória sobre a razão de probabilidade do evento morte. Para a variável Classe Diamétrica, o valor da estimativa de máxima verossimilhança é dado por $\mathrm{e}^{-0,4461}$, o que resulta em 0,640 (0,544 e 0,754; limites de confiança de Wald $(95 \%)$ ), significando que árvores com menor diâmetro têm 1,5625 vezes $(1 / 0,640)$ ou $156,25 \%$ de chances de morrer em relação às de maior diâmetro. Para a variável Copa, o valor da estimativa de máxima verossimilhança corresponde a $\mathrm{e}^{-0,4376}$, que resulta em $0,646(0,584$ e 0,713 ; limites de confiança de Wald (95\%)), mostrando que árvores com copa mais atingida pelo fogo têm 1,5480 vezes $(1 / 0,646)$ ou $154,80 \%$ de possibilidade de morrer em relação às menos atingidas. A estimativa de máxima verossimilhança para o parâmetro Casca não foram significativos $(p>0,05)$. A associação entre probabilidades previstas $\mathrm{e}$ respostas observadas mostram $72,1 \%$ de valores concordantes, $22,7 \%$ de discordantes e $5,2 \%$ de neutros, o que indica um bom ajuste do modelo.

No monitoramento da brotação (níveis I e II) foi verificada predominância absoluta desta ocorrência nas árvores de menor porte. No Nível I a maioria (cerca de $90 \%$ ) dos indivíduos com bro- tação possuem DAP menor do que $10 \mathrm{~cm}$, sendo que os dois indivíduos de maior porte encontrados foram com DAP de $44 \mathrm{~cm}$ e $21 \mathrm{~cm}$. O Nível II, portanto, apresentou taxas de brotação superiores ao Nível I, cerca de 3-4 vezes maior. Foi observada tendência de diminuição, ou mortalidade, da brotação ao longo das avaliações efetuadas em ambos os níveis de abordagem, sendo que os percentuais de indivíduos com brotação variaram entre $6,5 \%$ e $4,8 \%$ no Nível I e entre $26,3 \%$ e $16,5 \%$ no Nível II, respectivamente para a $2^{\mathrm{a}}$ avaliação (maio de 2006) e a $5^{\mathrm{a}}$ avaliação (janeiro de 2009).

Entre as formas de vida estudadas, foi observado que as espécies de palmeiras, seguidas das árvores lenhosas e dos cipós, são as que apresentam maior resiliência aos impactos do fogo. As palmeiras apresentaram, no decorrer do monitoramento, no Nível I, percentuais totais de indivíduos sem dano aparente (5-Não atingido) variando de $5,3 \%$ ( $1^{\text {a }}$ avaliação) a $61,6 \%$ ( $5^{\text {a }}$ avaliação), enquanto que as árvores lenhosas variaram de 2,1\% a $25,9 \%$ e os cipós de $4,3 \%$ a $21,4 \%$, respectivamente entre a $1^{\mathrm{a}}$ e a $5^{\mathrm{a}}$ avaliação. Da mesma forma, quanto aos danos máximos (mortalidade e indivíduos suprimidos), os menores índices de danos são a favor das palmeiras, seguidas das árvores e dos cipós. Os percentuais totais de indivíduos mortos (1-Desvitalizado) somados aos suprimidos (Sem informação), por exemplo, no Nível I, para as três formas de vida e para a $1^{\mathrm{a}}$ e a $5^{\mathrm{a}}$ avaliação, foram respectivamente: palmeiras $11,9 \% \mathrm{e}$ $37,0 \%$; árvores lenhosas $31,2 \%$ a $61,7 \%$; e cipós $36,8 \%$ a $74,4 \%$ (Figura 4 ). 


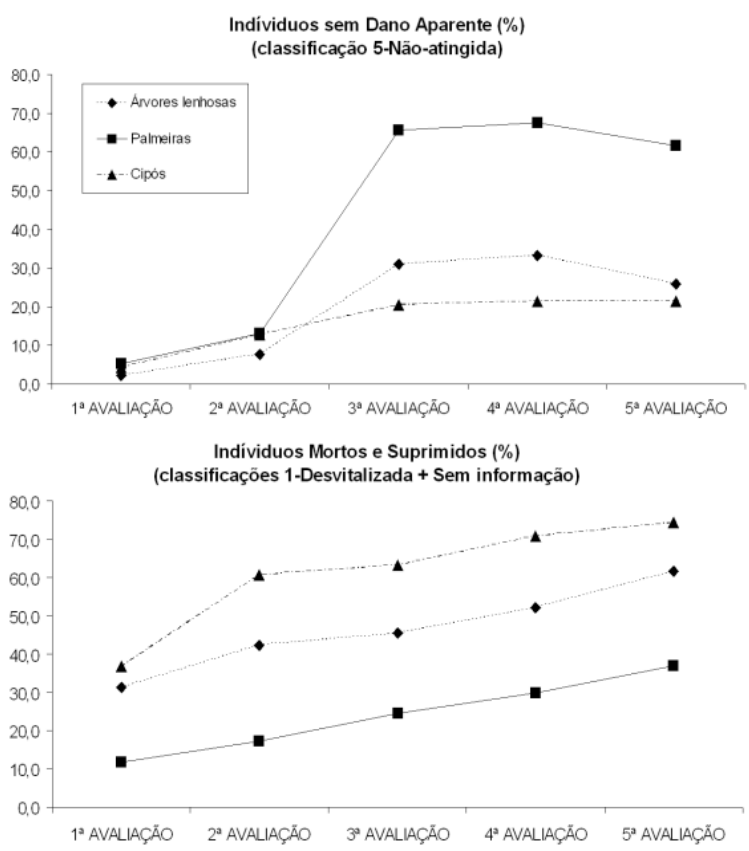

FIGURA 4: Percentuais de indivíduos sem dano aparente e dos mortos e suprimidos no Nível I de abordagem para árvores lenhosas, palmeiras e cipós.

FIGURE 4: Percentage of individuals with no apparent damage and the dead and the lost ones in Level I of tree size for trees, palm trees and lianas.

\section{CONCLUSÕES}

Os resultados deste estudo mostraram uma relação inversa entre o porte dos indivíduos (classe diamétrica) e os efeitos danosos do fogo incidente, ou seja, quanto menor o tamanho dos indivíduos maiores foram os impactos e as taxas de mortalidade sobrevindos, e vice-versa. Isto é constatado pelos percentuais crescentes do número de indivíduos dados como mortos (soma das classificações 1-Desvitalizado e Sem informação) na $5^{\mathrm{a}}$ avaliação do Nível II $(5 \mathrm{~cm}>\mathrm{DAP} \geq 2 \mathrm{~cm})$, que foi de $80,1 \%$, nas menores classes diamétricas do Nível I $(5 \mathrm{~cm} \leq$ DAP $<10 \mathrm{~cm}$ e $10 \mathrm{~cm} \leq$ DAP $<30 \mathrm{~cm}$ ), que foram de $71,4 \%$ e $48,3 \%$, respectivamente, e pelos percentuais nulos $(0 \%)$ de mortalidade das classes diamétricas superiores do Nível I $(70 \mathrm{~cm} \leq$ DAP $<90 \mathrm{~cm}$ e DAP $\geq 90 \mathrm{~cm})$. As análises estatísticas confirmam essa assertiva.

Contudo, houve de modo generalizado, embora não tenha sido notado entre a $4^{\mathrm{a}}$ e a $5^{\mathrm{a}}$ avaliação, em todas as classes diamétricas (níveis I e II), inclusive nas avaliações da Casca e Copa, um crescimento acelerado e contínuo do número de indivíduos classificados sem dano aparente (5-Não atingido) e, além disso, houve aumento de $43 \%$ no número de espécies da regeneração (Nível III).

$\mathrm{O}$ crescimento do número de indivíduos sem dano aparente e do número de espécies da regeneração pode ser interpretado como indicativo de um processo de recuperação, ou revegetação, da floresta frente ao fogo. No entanto, com base nos padrões de danos causados apenas por um incêndio, que é o caso da área estudada, é esperado que a incidência de novos incêndios, em curtos intervalos insuficientes à recuperação, resulte na irremediável degeneração da floresta.

A respeito de modificações na composição florística original, os dados de redução da diversidade observados (15\% no Nível I e 33 \% no Nível II) são preocupantes, pois, embora restritos às áreas das parcelas amostrais (1,6 ha do Nível I e 0,4 ha do Nível II) e não a toda área do estudo, além de relativos aos indivíduos jovens e adultos e não à regeneração e ao banco de sementes, permitem inferir que a floresta em conjunto pode ter sido seriamente afetada em relação a esse aspecto.

\section{REFERÊNCIAS BIBLIOGRÁFICAS}

ALENCAR, A. et al. Desmatamento na Amazônia: indo além da emergência crônica. Manaus, Instituto de Pesquisa Ambiental da Amazônia (IPAM), 2004, 89 p.

BALCH, J. K. et al. Negative fire feedback in a transitional forest of southeastern Amazonia. Global Change Biology, v. 10, p. 2276-2287. 2008. BARLOW, J.; PERES, C. A. Fogo Rasteiro: nova ameaça na Amazônia. Ciência Hoje. São Paulo: v. 199, p. 24-29. 2003.

BRANDO, P. M. et al. Drought effects on litterfall, wood production and belowground carbon cycling in an Amazon forest: results of a throughfall reduction experiment. Philosophical Transactions of the Royal Society B, v. 1498, p. 1839-1848. 2008.

BRASIL. Ministério das Minas e Energia. Departamento de Produção Mineral. Projeto Radambrasil. Folha SC19. Levantamento dos Recursos Naturais. V. 12. Rio Branco. Rio de Janeiro, RJ. 1976. 458 p.

BROWN, I. F. etal. Monitoring Fires in Southwestern Amazonia Rain Forests. EOS Transactions. American Geophysical Union, v. 87, p. 253-264. 2006.

COX, P. M. et al. Acceleration of global warming 
due to carbon cycle feedbacks in a coupled climate model. Nature, v. 408, p. 184-187. 2000.

COX, P. M. et al. Increasing risk of Amazonian drought due to decreasing aerosol pollution. Nature, v. 453, p. 212-215. 2008.

FEARNSIDE, P. M. A floresta Amazônia nas mudanças globais. Manaus, Instituto Nacional de Pesquisas da Amazônia (INPA). 2003. 134 p.

FERREIRA, L. V. et al. O desmatamento na Amazônia e a importância das áreas protegidas. Estudos Avançados, São Paulo, v. 53, p. 157-166. 2005.

IBGE. Instituto Brasileiro de Geografia e Estatística. Indicadores de desenvolvimento sustentável: Brasil 2008. Rio de Janeiro. 2008. 471 p. LAURANCE, W. L. et al. Deforestation in Amazonia. Science, v. 304, p. 1109-1111. 2004.

MAPA. Ministério da Agricultura, Pecuária e Abastecimento. Agritempo - Sistema de Monitoramento Agrometeorológico. Embrapa Informática Agropecuária. Disponível em: $<$ (http://www.agritempo.gov.br/agroclima/ sumario? $\mathrm{uf}=\mathrm{AC})>$. Acesso em: 24 de junho de 2009.

MARENGO, J. A. et al. Hydro-climatic and ecological behavior of the drought of Amazonia in
2005. Philosophical Transactions of the Royal Society B, v. 363, p. 1773-1778. 2008b.

MARENGO, J. A. et al. The drought of Amazonia in 2005. Journal of Climate. v. 21, p. 495-516. $2008 \mathrm{a}$.

MARGULIS, S. Causas do Desmatamento da Amazônia Brasileira. Banco Mundial. 1. Ed. Brasília. 2003. 100 p.

NEPSTAD, D. et al. A floresta em chamas: origens, impactos e prevenção de fogo na Amazônia. 1. ed. Brasília: IPAM. 1999. 202 p.

NEPSTAD, D. et al. Amazon drought and its implications for forest flammability and tree growth: a basin-wide analysis. Global Change Biology, v. 10, p. 704-717. 2004.

NEPSTAD, D. et al. The effects of partial throughfall exclusion on canopy processes, aboveground production, and biogeochemistry of an Amazon forest. Journal of Geophysical Research -Atmospheres, v. 107, p. 80-85. 2002.

NOBRE, C. A. et al. Mudanças climáticas e Amazônia. Ciência e Cultura. São Paulo, v. 3, p. 22-27. 2007.

PHILLIPS, O. L. et al. Drought Sensitivity of the Amazon Rainforest. Science, v. 5919, p. 1344 1347. 2009. 